1.

\title{
BECKETT: A QUINTESSÊNCIA DA AUTOTRADUÇÃO?
}

\section{Rainier Grutman}

University of Ottawa

Este artigo propõe uma tipologia de autotradutores a partir do famoso caso de Samuel Beckett, que tem sido muitas vezes visto como o escritor bilíngue por excelência. Infelizmente, tal posição é um obstáculo a uma compreensão melhor e mais geral da autotradução, uma prática da qual o escritor irlandês, vencedor do Prêmio Nobel, é apenas um exemplo não muito típico, apesar de fascinante. Beckett, em minha opinião, pode ajudar-nos a ganhar muitos insights sobre a autotradução, mas somente se nos permitirmos olhar para além dele, em vez de ficar na sombra (reconhecidamente longa) que ele lança.

Palavras-chave: autotradução, tipologia, autotradutores

\section{BECKETT: THE QUINTESSENCE OF SELF-TRANSLATION?}

This essay proposes a typology of self-translators starting from the rightly famous case of Samuel Beckett, who has often been constructed as the quintessential bilingual writer. Unfortunately, this situation stands in the way of a better, more general comprehension of self-translation as such, a practice of which the Irish Nobel laureate's balancing act, no matter how fascinating, is but one example and not a very typical one at that. Beckett, I argue, can indeed help us gain many insights into self-translation, but only if we allow ourselves to look beyond him, instead of staying in the (admittedly long) shadow he casts.

Keywords: self-translation, tipology, self-translators

2. A DECISÃO DE TRADUZIR O PRÓPRIO TEXTO, MOTIVAÇÕES E
CONSEQUÊNCIAS: UM BREVE ESTUDO DOS CASOS DOS ESCRITORES
BRASILEIROS ANA MARIA MACHADO E JOÃO UBALDO RIBEIRO

Maria Alice Antunes

Universidade do Estado do Rio de Janeiro / UERJ

Bianca Walsh

Universidade do Estado do Rio de Janeiro / UERJ

Este artigo trata das razões e consequências da autotradução. Essa opção pode ter vários motivos: o desejo de atingir mais leitores, o de controlar o texto, o exílio, a censura. As consequências, contudo, são pouco exploradas. Discutiremos as razões e as consequências dessa opção para a obra, a carreira no sistema literário de origem e no sistema alvo, e para o público leitor, com base nos casos de Ana Maria Machado e João Ubaldo Ribeiro. Esperamos contribuir para o preenchimento de "espaços em branco" (Santoyo, 2004) nos Estudos da Tradução.

Palavras-chave: autotradução; razões; consequências 


\section{THE DECISION TO TRANSLATE ONE'S OWN TEXT, REASONS AND CONSEQUENCES: A STUDY OF THE CASES OF THE BRAZILIAN WRITERS ANA MARIA MACHADO AND JOÃO UBALDO RIBEIRO}

This article presents the reasons and consequences of self-translation. This choice may be due to various reasons: the desire to reach a wider audience, the attempt to control the text, exile, censorship. The consequences, however, have not been widely explored. We will discuss the reasons and consequences of that option for an author's work, for his career in the literary system of origin and in the target literary system, and for readers. Our discussion will focus on the cases of Ana Maria Machado and João Ubaldo Ribeiro. We want to contribute for filling in the "blank spaces" (Santoyo, 2004) in Translation Studies.

Keywords: self-translation; reasons; consequences

3.

"LOREM IPSUM": UMA AUTOVERSÃO POÉTICA

Paulo Henriques Britto

PUC-Rio

Neste artigo, analisa-se a tradução de um poema escrito em português, língua nativa do autor, para o inglês, seu segundo idioma, feita pelo próprio autor. 0 poema original e sua tradução são analisados em termos de seus componentes fonológicos, esquemas de rima e de métrica, bem como no plano do significado. Comparando-se as duas análises, conclui-se que a proximidade entre os dois textos permite a afirmação de que se trata de uma tradução propriamente dita.

Palavras-chave: tradução de poesia, autotradução, versão

\section{“LOREM IPSUM": POETIC SELF-TRANSLATION INTO A SECOND LANGUAGE}

The article is an analysis of a poem written in Portuguese by a native speaker of Portuguese and its translation, by the author, into English, his second language. Original and translation are analyzed as to their phonological components, rhyme schemes and meter, as well as meaning. Comparison between the two analyses shows that the two texts are close enough to be thought of as representing a case of translation proper.

Keywords: poetry translation, self-translation, translation into a second language

4.

\section{RE-AUTOTRADUCCIÓN. EL CASO DE PICCOLO KARMA DE CARLO COCCIOLI Valentina Mercuri}

AUTOTRAD, Universidad Autónoma de Barcelona

Resumen:

El caso extremo de autotraducción de Piccolo Karma del escritor italiano Carlo Coccioli y en concreto la re-autotraducción del texto español nos hace reflexionar sobre la influencia del factor temporal, y por tanto de la memoria, en la reedición 
del texto autotraducido. Asimismo, tomamos en consideración la direccionalidad de la autotraducción como elemento que determina los cambios aportados en la segundo edición de PK. Del análisis comparativo se ha sacado a relucir la hipercorrección que el autor-traductor realiza al volver sobre su traducción, empujado por un afán de perfeccionismo.

Palavras clave: re-autotraducción, hipercorrección, perfeccionismo

\section{RE-AUTOTRADUÇÃO. O CASO DE PICCOLO KARMA DE CARLO COCCIOLI}

O exemplo extremo da autotradução de Piccolo Karma do escritor italiano Carlo Coccioli e, em particular, a re-autotradução do texto espanhol nos faz refletir sobre a influência do fator tempo, e portanto da memória, na reedição do texto autotraduzido. Ainda assim, levamos em consideração a direção da autotradução como elemento que determina as mudanças acrescentadas na segunda edição de PK. Da análise comparativa veio à tona a hipercorreção que o autor-tradutor realiza ao retomar sua própria tradução, impulsionado por um desejo de perfeição.

Palavras-chave: re-autotradução, hipercorreção, perfeição

5.

ANTONIO D'ALFONSO OU DU VERTIGE AUTOTRADUCTIF

Alessandra Ferraro

Université de Udine

\section{Résumé}

Antonio D’Alfonso (1953) est l'un des auteurs canadiens qui, en conséquence de leur éducation transculturelle, ont constamment pratiqué l'autotraduction. Dans cet article, nous présentons une étude du recueil poétique The Other Shore / L'autre rivage, qui comprend des autotraductions de poèmes écrits à l'origine dans l'autre langue. Par le biais de l'autotraduction, D'Alfonso essaie de trouver un moyen pour réconcilier les différentes facettes de son moi. Cependant, il s'agit d'un travail toujours inachevé car les deux langues acquises, le français et l'anglais, ne sont pas en mesure d'exprimer le vide provoqué par l'absence du dialecte molisan, sa langue maternelle. Ce manque pousse le poète à constamment s'autotraduire à la recherche d'une langue mythique où toutes les langues résonnent d'une façon mélodieuse.

Mots-clés: autotraduction, poème, langue mythique

\section{ANTONIO D'ALFONSO: VERTIGEM AUTOTRADUTORIA}

Antonio D'Alfonso (1953) é um dos autores canadenses que, em consequência de sua educação transcultural, praticou a autotradução com frequência. Neste artigo, apresentamos um estudo sobre a coletânea de poemas The Other Shore / L'autre rivage, que inclui as autotraduções de poemas escritos originalmente na outra língua. Através da autotradução, D’Alfonso tenta achar meios para reconciliar as diferentes facetas de seu eu. Contudo, é um trabalho sempre inacabado porque ambas as línguas adquiridas, o francês e o inglês, não são capazes de expressar o 
vácuo causado pela ausência do dialeto Molisan, sua língua materna. Essa falta empurra o poeta a autotraduzir-se de maneira constante em busca de uma língua mítica, em que todas as línguas ressoem de forma melodiosa.

Palavras-chave : autotradução, poema, língua mítica

6.

DE L'ECRITURE A L'AUTOTRADUCTION. L'HISTOIRE MIGRANTE DE GENS DU SILENCE DE MARCO MICONE

Paola Puccini

Université de Bologne

\section{Résumé}

L'autotraduction chez Marco Micone donne à voir un espace de réélaboration des origines et de l'œuvre elle-même qui, en passant entre les langues et en réfléchissant à la complexité de l'appartenance identitaire, trouve une nouvelle forme d'expression. Nous nous proposons de suivre l'itinéraire de Gens du silence, de 1982, qui connait deux versions successives, la première en 1991 et la deuxième en 1996. C'est cette dernière version que l'auteur traduira une première fois en italien (Non era per noi, janvier 2004) et presque simultanément une deuxième fois en français (Silences, mars 2004). En nous interrogeant sur le résultat de cette opération autotraductive, nous retracerons les étapes du rapprochement de l'auteur de sa langue et de sa culture d'origine en montrant le travail de réélaboration de ses rapports avec les origines. Grâce à cette expérience, Micone travaille simultanément son rapport aux origines en faisant coincider « origine» et «original ». Libre de se traduire sans craindre d'être infidèle, il découvre une égale liberté dans le travail de déconstruction et de reconstruction de sa mémoire imaginée, réinventée et « autotraduite ».

Mots-clés: ecriture, autotraduction, origine, original

\section{DA ESCRITA À AUTOTRADUÇÃO. A HISTÓRIA MIGRANTE DE GENS DU SILENCE DE MARCO MICONE}

A autotradução em Marco Micone apresenta um espaço de recriação das origens e da própria obra que, ao passar entre as línguas e ao refletir a complexidade do sentimento de pertença, acha uma nova forma de expressão. Propomo-nos a seguir a rota de Gens du silence de 1982 que tem duas versões sucessivas, a primeira em 1991 e a segunda em 1996. A última versão foi traduzida pelo autor, uma primeira vez para o italiano (Non era per noi, janeiro de 2004) e, quase simultaneamente, uma segunda vez para o francês (Silences, março de 2004). Ao investigar o resultado da autotradução, refaremos os passos da reconciliação do autor com sua língua e sua cultura de origem mostrando o trabalho de reelaboração de sua ligação às origens. Graças a essa experiência, Micone trabalha simultaneamente sua ligação às origens ao fazer coincidir a " origem » e o " original ». Livre para traduzir-se sem medo de ser infiel, o autor descobre igual liberdade no trabalho de descontrução e de reconstrução de sua memória imaginada, reinventada e «autotraduzida ». 
Palavras-chave: escrita, autotradução, origem, original

7.

\author{
AUTOTRADUCTION ET FIGURES DU DÉDOUBLEMENT DANS LA \\ PRODUCTION DE NANCY HUSTON \\ Valeria Sperti \\ Université de la Basilicate
}

\begin{abstract}
Résumé
L'article illustre le parcours linguistique et d'autotraduction de Nancy Huston pour montrer que les polémiques suscitées par l'attribution du prix du Gouverneur général du Canada à la version française de Plainsong (1993) ont profondément influencé sa production littéraire et essayistique. Romain Gary et Samuel Beckett deviennent ses génies tutélaires : les essais que Nancy Huston leur consacre entre 1995 et 2000 revendiquent l'ambiguïté irréductible de leur in-between linguistique, qui est aussi celle de l'écrivaine. Ils montrent aussi une troisième voie, où les langues, et les dédoublements qui en dérivent, ne seraient plus en opposition, mais en compréhension réciproque, l'une acceptant l'autre. Cette position est examinée dans Instruments des ténèbres (1996) où les dédoublements narratifs, linguistiques et textuels sont analysés comme autant d'autobiographèmes, montrant que l'auteure incarne dans des personnages romanesques ses propres doubles linguistiques et identitaires. L'article se termine sur quelques considérations sur l'évolution des configurations du dédoublement et de l'autotraduction dans Lignes de faille (2005) et Danse noire (2013).
\end{abstract}

Mots-clés: autotraduction, in-between linguistique, dédoublements

\title{
AUTOTRADUÇÃO E FIGURAS DE DUPLICIDADE NA PRODUÇÃO DE NANCY HUSTON
}

$\mathrm{O}$ artigo ilustra o percurso linguístico e autotradutório de Nancy Huston para mostrar que as polêmicas causadas pela atribuição do prêmio do Governador-geral do Canadá à versão francesa de Plainsong (1993) influenciaram de maneira profunda sua produção literária e ensaística. Romain Gary e Samuel Beckett tornam-se seus gênios inspiradores: os ensaios que Nancy Huston lhes dedica entre 1995 e 2000 reivindicam seu entrelugar linguístico, que é também o da escritora. Os ensaios também mostram uma terceira via, onde as línguas e a consequente duplicidade não estariam em oposição, mas em compreensão mútua, uma aceita a outra. Essa posição é examinada em Instruments des ténèbres (1996) onde as duplicidades narrativa, linguística e textual são analisadas como autobiographèmes, mostrando que a autora encarna nos personagens fictícios sua própria duplicidade linguística e identitária. $\mathrm{O}$ artigo termina com algumas considerações sobre a evolução das configurações da duplicidade e da autotradução em Lignes de faille (2005) et Danse noire (2013).

Palavras-chave: autotradução, entrelugar linguístico, duplicidade

8.

HUSTON, WE HAVE A PROBLEM... (OR WHAT ON EARTH IS “CANADIAN SELF-TRANSLATION" SUPPOSED TO MEAN?)

Trish Van Bolderen 
University of Ottawa

This article focuses on the conceptual and methodological problems that Nancy Huston poses for research into Canadian self-translation. A Canadian citizen by birthright, Huston has lived in France for decades, producing all of her self-translations there; yet scholars seem ready to classify her alongside writers who have self-translated in Canada. I illustrate how Huston's Canadianness is perceived, contrasting this identity with that of 16 other self-translators. Adopting a sociological approach, I underscore the challenges and importance of defining criteria for national belonging, arguing that, while Huston may be a self-translator who is Canadian, she is not a Canadian selftranslator.

Keywords: self-translation, Canada, national belonging

\section{HUSTON, TEMOS UM PROBLEMA... (OU QUE DIABOS SIGNIFICA “AUTOTRADUÇÃO” CANADENSE?)}

Este artigo enfoca os problemas conceituais e metodológicos que Nancy Huston representa para a pesquisa acerca da autotradução canadense. Cidadã canadense por nascimento, Huston vive na França há décadas, produzindo todas as suas autotraduçōes no país. No entanto, estudiosos prontamente a classificam ao lado de escritores que têm autotraduzido no Canadá. Procuramos ilustrar como o canadianismo de Huston é concebido, contrastando essa identificação com a de outros 16 autotradutores. Adotando uma visão sociológica, salientamos os desafios e a importância da definição de critérios para reconhecimento nacional, argumentando que, enquanto Huston pode ser autotradutora e canadense, ela não é uma autotradutora canadense.

Palavras-chave: autotradução, Canadá, reconhecimento nacional

9.

ITALIAN META-REFLECTIONS ON SELF-TRANSLATION: AN OVERVIEW OF THE DEBATE

Tiziana Nannavecchia

University of Ottawa

In 2010, the first two events entirely dedicated to self-translation took place in Udine and Pescara, Italy. Since then, four international conferences have been held, demonstrating the willingness to maintain a dialogue on a topic that is far from being exhausted or fully understood. Four out of six gatherings took place in Italy; for this reason, and for the subsequent publication in that country of three volumes containing the proceedings of as many conferences, Italy represents a fertile territory to examine the current state of the debate.

Keywords: self-translation, debate, Italy

\section{META-REFLEXÕES ITALIANAS SOBRE A AUTOTRADUÇÃO: UMA VISÃO GERAL DO DEBATE}

Em 2010, os dois primeiros eventos inteiramente dedicados a autotradução aconteceram em Udine e Pescara, na Itália. Desde então, quatro conferências internacionais foram 
realizadas. Tal fato demonstra o desejo de diálogo sobre um tema que não foi exaurido ou compreendido totalmente. Quatro de seis reuniões aconteceram na Itália. Por essa razão, e pela subsequente publicação naquele país dos anais de outras conferências, a Itália representa um território fértil para o exame do estado atual do debate.

Palavras-chave: autotradução, debate, Itália 